\title{
Impact of inflammatory markers on survival in patients with limited disease small-cell lung cancer undergoing chemoradiotherapy
}

This article was published in the following Dove Press journal:

Cancer Management and Research

\section{Denise Bernhardt, ${ }^{1-3, *}$ \\ Sophie Aufderstrasse, 1,2,* Laila König, ${ }^{1-3}$ Sebastian Adeberg, ${ }^{1-4}$ Farastuk Bozorgmehr, ${ }^{5,6}$ Petros Christopoulos, ${ }^{5,6}$ Rami A El Shafie, ${ }^{1,2}$ Juliane Hörner- Rieber, ${ }^{1-3}$ Jutta Kappes, ${ }^{6,7}$ Michael Thomas, ${ }^{5,6}$ Felix Herth, ${ }^{6,7}$ Martin Steins, ${ }^{5}$ Jürgen Debus, ${ }^{1-4}$ Stefan Rieken $^{1-3}$ \\ 'Department of Radiation Oncology, University Hospital Heidelberg, Heidelberg, Germany; ${ }^{2}$ Heidelberg Institute of Radiation Oncology (HIRO), Heidelberg, Germany; ${ }^{3}$ Heidelberg lon-Beam Therapy Center (HIT), Heidelberg, Germany; ${ }^{4}$ Clinical Cooperation Unit Radiation Oncology, German Cancer Research Center (DKFZ), Heidelberg, Germany; ${ }^{5}$ Department of Thoracic Oncology, Thoraxklinik, Heidelberg University, Translational Lung Research Centre Heidelberg (TLRC-H), Heidelberg, Germany; ${ }^{6}$ German Centre for Lung Research (DZL), Heidelberg, Germany; ${ }^{7}$ Department of Pneumology, \\ Thoraxklinik, Heidelberg University, Heidelberg, Germany \\ *These authors contributed equally to this work}

Correspondence: Denise Bernhardt Department of Radiation Oncology, University Hospital Heidelberg, Im Neuenheimer Feld 400, Heidelberg 69120, Germany

Tel +4962 21568203

Email denise.bernhardt@med.uniheidelberg.de
Background: Systemic inflammation appears to play a role in the progression of numerous solid tumors by promoting tumor proliferation. Our current study aimed to evaluate the role of inflammatory markers in limited disease (LD) small-cell lung cancer (SCLC) patients undergoing thoracic chemoradiotherapy (TCR).

Patients and methods: We retrospectively analyzed a total number of 350 SCLC patients diagnosed with LD SCLC who received TCR between 1999 and 2017 and had available blood tests within 2 weeks prior to the start of TCR. Serum C-reactive protein (CRP), neutrophil-tolymphocyte ratio (NLR), lactate dehydrogenase (LDH), hemoglobin (Hb) levels, and platelet count $(\mathrm{Pc})$ were evaluated as potential inflammatory markers. Kaplan-Meier survival analysis was performed for overall survival (OS). For comparison of survival curves, the log-rank (Mantel-Cox) test was used. Univariate and multivariate Cox proportional HRs were used to assess the influence of cofactors on OS.

Results: Univariate analysis for OS revealed a statistically significant effect for $\mathrm{LDH}>400 \mathrm{U} / \mathrm{L}$ (HR $2.05 \mathrm{U} / \mathrm{L} ; 95 \%$ CI 1.29-3.26 U/L; $P=0.002$ ), prophylactic cranial irradiation (PCI; HR 0.58; 95\% CI 0.40-0.85; $P=0.005)$, CRP > 50 mg/L (HR 1.49 mg/L; 95\% CI 1.05-2.10 mg/L; $P=0.026$ ), and Karnofsky performance scale (KPS) $<70 \%$ (HR $1.35 \%$; 95\% CI $1.02-1.80 \%$; $P=0.035)$. NLR, age ( $>70$ years), Hb levels, and Pc did not influence survival. In multivariate analysis, OS was significantly affected by PCI (HR 0.64; 95\% CI $0.43-0.94 ; P=0.026$ ), LDH >400 U/L (HR 1.91 U/L; 95\% CI 1.21-3.05 U/L; $P=0.006$ ), and CRP > 50 mg/L (HR 1.43 mg/L; 95\% CI $1.01-2.04 \mathrm{mg} / \mathrm{L} ; P=0.045)$. KPS $(\leq 70 \%)$ did not influence survival in multivariate analysis. Conclusion: Elevated CRP and LDH seem to be the independent prognostic factors for OS in LD SCLC patients undergoing TCR. However, elevated NLR was not found to be an independent prognostic factor for OS if taken prior to TCR. LDH and CRP are easily available blood tests and do not require additional resources for routine use and could be useful for clinical decision making.

Keywords: small-cell lung cancer, limited disease, comorbidity, NLR, LDH, CRP

\section{Introduction}

Systemic inflammation is increasingly being recognized as an important prognostic factor of survival in cancer patients, and several markers of systemic inflammation, such as C-reactive protein (CRP) and lactate dehydrogenase (LDH), were investigated for their potential impact on outcomes. ${ }^{1-5}$ Furthermore, several studies suggested that neutrophil-to-lymphocyte ratio (NLR) had a prognostic value in predicting the survival of patients with solid tumors. ${ }^{6-14}$ As nonspecific inflammatory markers, previous research found that the elevation of pretreatment NLR, LDH, and CRP was 
negatively associated with survival in lung cancer patients. However, the results of individual studies are inconsistent, particularly between histological subtypes and disease stage. Currently, there are several analyses available for non-smallcell lung cancer (NSCLC) subtypes, but the data on SCLC patients are inconsistent with small cohorts, inhomogeneous treatments, or mixed disease stage. ${ }^{7-9,12,14-18}$ Moreover, NLR cutoff values are yet determined to be safely used for clinical decision making.

This study assessed the prognostic value of the pretreatment inflammatory markers in limited disease (LD) SCLC patients undergoing thoracic chemoradiotherapy (TCR).

\section{Patients and methods}

\section{Patients and treatment features}

We identified 350 patients in our cancer center database who had histologically confirmed LD SCLC according to the classification of the Veterans Affairs Administration Lung Cancer Study Group (VALG) ${ }^{19}$ between 1999 and 2017, which received standard concurrent TCR as a first-line treatment. Blood tests within 2 weeks prior to the start of TCR were retrospectively used for analysis. Serum CRP, NLR, $\mathrm{LDH}$, hemoglobin $(\mathrm{Hb})$ levels, and platelet count $(\mathrm{Pc})$ were evaluated as potential inflammatory markers. Thoracic RT was administered once daily (one daily fraction of 2 Gy on 5 consecutive days a week), concurrently with chemotherapy. Patients with complete remission (CR) or good partial response $(\mathrm{PR})$ after initial therapy received prophylactic cranial irradiation (PCI). The detailed characteristics of the patients are given in Table 1. All reviews were performed following institutional guidelines and the Declaration of Helsinki 1975 in its most recent version. Ethical approval for the study and a waiver of written informed consent were obtained from the local ethics committee, University

Table I Characteristics of the patients

\begin{tabular}{|l|l|}
\hline Variable & $\mathbf{N}=\mathbf{3 5 0}$ \\
\hline Median age (range), years & $64(37-93)$ \\
\hline Median KPS \% (range) & $80(50-100)$ \\
\hline Male, $\mathrm{n}(\%)$ & $213(6 \mathrm{I})$ \\
\hline Female, $\mathrm{n}(\%)$ & $137(39)$ \\
\hline $\mathrm{PCl}, \mathrm{n}(\%)$ & $299(85)$ \\
\hline Median LDH (range), U/L & $248(123-\mathrm{I}, \mathrm{I36})$ \\
\hline Median CRP (range), $\mathrm{mg} / \mathrm{L}$ & $28(<\mathrm{I}-280)$ \\
\hline Median Pc (range), /nL & $30 \mathrm{I}(4-909)$ \\
\hline Median Hb (range), g/dL & $13.8(8-18)$ \\
\hline Median NLR (range) & $4(0.5-18.5)$ \\
\hline
\end{tabular}

Abbreviations: KPS, Karnofsky performance scale; $\mathrm{PCl}$, prophylactic cranial irradiation; LDH, lactate dehydrogenase; CRP, C-reactive protein; Pc, platelet count; $\mathrm{Hb}$, hemoglobin; NLR, neutrophil-to-lymphocyte ratio.
Hospital Heidelberg (\#S-461/2016). Patient confidentiality was maintained by anonymizing patient data to remove any identifying information.

\section{Statistical analyses}

A statistical analysis was carried out using SigmaPlot ${ }^{\mathrm{TM}}$ (Systat Software GmbH, Erkrath, Germany). Univariate Cox proportional HRs were used to assess the influence of cofactors on overall survival (OS). For comparison of survival curves, log-rank (Mantel-Cox) test was used. OS was defined as the time from the first fraction of radiotherapy to death. Living patients were censored from survival analysis at last known contact. A $P$ value of $<0.05$ was considered as statistically significant. NLR was calculated by dividing the number of neutrophils by lymphocyte number. NLR cutoff values of $>4$ and $>2.65$ were used to compare the results with previous findings. ${ }^{11,20}$

\section{Results}

Univariate analysis for OS revealed a statistically significant effect for LDH $>400$ (HR 2.05; 95\% CI 1.29-3.26; $P=0.002$ ), PCI (HR 0.58; 95\% CI 0.40-0.85; $P=0.005), \mathrm{CRP}>50$ (HR $1.49 ; 95 \%$ CI $1.05-2.10 ; P=0.026)$, and Karnofsky performance scale (KPS) $<70 \%$ (HR $1.35 ; 95 \%$ CI $1.02-1.80$; $P=0.035)$. NLR, age ( $>70$ years), $\mathrm{Hb}$, and Pc did not influence survival (Table 2).

In multivariate analysis, OS was significantly affected by PCI (HR 0.64; 95\% CI 0.43-0.94; $P=0.026$ ), LDH $>400$ (HR $1.91 ; 95 \%$ CI 1.21-3.05; $P=0.006)$, and CRP $>50$ (HR 1.43; $95 \%$ CI 1.01-2.04; $P=0.045)$. KPS ( $\leq 70 \%)$ did not influence survival in multivariate analysis (Table 3 ).

The median OS calculated from the first day of RT was 20 months. Patients who received PCI had a significantly longer survival compared to patients who did not receive PCI

Table 2 Univariate proportional hazards regression analysis of cofactors associated with OS

\begin{tabular}{|l|l|l|l|l|}
\hline \multicolumn{2}{|l|}{ Factors analyzed } & HR & $\mathbf{9 5 \%} \mathbf{C l}$ & $\boldsymbol{P}$ \\
\hline $\mathrm{NLR}$ & $<2.65$ & 0.86 & $0.64-\mathrm{I} .15$ & 0.32 \\
\hline $\mathrm{NLR}$ & $<4$ & 0.92 & $0.7 \mathrm{I}-\mathrm{I} .19$ & 0.52 \\
\hline $\mathrm{LDH}$ & $>400 \mathrm{U} / \mathrm{L}$ & $\mathbf{2 . 0 5}$ & $\mathrm{I} .29-3.26$ & $\mathbf{0 . 0 0 2}$ \\
\hline $\mathrm{Hb}$ & $\mathrm{g} / \mathrm{dL}$ & 0.97 & $0.89-1.05$ & 0.49 \\
\hline $\mathrm{Pc}$ & $<125 / \mathrm{hL}$ & 0.5 & $0.16-1.63$ & 0.25 \\
\hline $\mathrm{CRP}$ & $>50 \mathrm{mg} / \mathrm{L}$ & $\mathbf{I . 4 9}$ & $\mathrm{I} .05-2.10$ & $\mathbf{0 . 0 2 6}$ \\
\hline Age, years & $>70$ & $\mathrm{I} .23$ & $0.93-1.63$ & 0.15 \\
\hline $\mathrm{PCl}$ & & $\mathbf{0 . 5 8}$ & $0.40-0.85$ & $\mathbf{0 . 0 0 5}$ \\
\hline KPS & $\leq 70 \%$ & $\mathbf{I . 3 5}$ & $\mathrm{I} .02-1.80$ & $\mathbf{0 . 0 3 5}$ \\
\hline
\end{tabular}

Note: Bold values indicate significant cofactors in univariate analysis.

Abbreviations: OS, overall survival; NLR, neutrophil-to-lymphocyte ratio; LDH, lactate dehydrogenase; $\mathrm{Hb}$, hemoglobin; Pc, platelet count; CRP, C-reactive protein; $\mathrm{PCl}$, prophylactic cranial irradiation; KPS, Karnofsky performance scale. 
(median 20 vs 12 months; $P=0.005$ ). Patients with a KPS $<70$ had a significantly shorter survival compared to patients with a KPS >70 (17 vs 20 months; $P=0.010$; Figure 1). Compared to patients with a CRP $<50$, patients with a CRP $>50$ had a significantly longer survival (median 20 vs 14 months; $P=0.025$; Figure 2). Furthermore, an LDH $>400$ was associated with a significantly shorter survival compared to an LDH $<400$ (median 13 vs 20 months; $P=0.008$; Figure 3 ).

\section{Discussion}

In the present study, we investigated the relationship between inflammatory markers, such as CRP, NLR, LDH, Hb, and Pc in patients with LD SCLC undergoing TCR. In univariate analysis, serum CRP level and LDH level were significantly

Table 3 Multivariate proportional hazards regression analysis of cofactors associated with OS

\begin{tabular}{|l|l|l|l|l|}
\hline \multicolumn{2}{|l|}{ Factors analyzed } & HR & $\mathbf{9 5 \%} \mathbf{C l}$ & $\boldsymbol{P}$ \\
\hline $\mathrm{LDH}$ & $>400 \mathrm{U} / \mathrm{L}$ & $\mathbf{I . 9 2}$ & $\mathbf{1 . 2 0 - 3 . 0 5}$ & $\mathbf{0 . 0 0 6}$ \\
\hline $\mathrm{CRP}$ & $>50 \mathrm{mg} / \mathrm{L}$ & $\mathbf{I . 4 3}$ & $\mathrm{I} .00-2.04$ & $\mathbf{0 . 0 4 5}$ \\
\hline $\mathrm{KPS}$ & $\leq 70 \%$ & 1.22 & $0.91-1.64$ & 0.184 \\
\hline $\mathrm{PCl}$ & Performed & $\mathbf{0 . 6 5}$ & $0.44-0.95$ & $\mathbf{0 . 0 2 6}$ \\
\hline
\end{tabular}

Note: Bold values indicate significant cofactors in multivariate analysis. Abbreviations: OS, overall survival; LDH, lactate dehydrogenase; CRP, C-reactive protein; KPS, Karnofsky performance scale; $\mathrm{PCl}$, prophylactic cranial irradiation. associated with OS. Among the inflammatory markers examined in this study, multivariate analysis revealed that only elevated serum CRP and LDH were predictors of survival. The relevance of pretreatment NLR and Pc noted in previous studies was not confirmed in univariate and multivariate analyses.

In the present study, NLR measured as a continuous variable (results not shown) as well as NLR $>4$ and $>2.65$ did not show a significant association with survival and contrasts previous results. ${ }^{11,20}$ The underlying mechanisms regarding the prognostic value of NLR are not fully understood but might reflect an increase in inflammation or a decrease in antitumor immunity. ${ }^{21}$ Neutrophils have been shown to be inductors of acute-phase proteins, including CRP, which has also been reported to predict poor prognosis in several tumor types. ${ }^{17,22-26}$ The present study revealed that elevated CRP is a strong predictor of outcome in LD patients undergoing TCR and confirmed previous studies, showing a significant correlations between elevated serum LDH levels and poor OS in patients with SCLC.

Inflammation plays an important role in tumor progression, and baseline immune suppression and systemic inflammation are associated with inferior survival. ${ }^{3,4}$ Inflammation

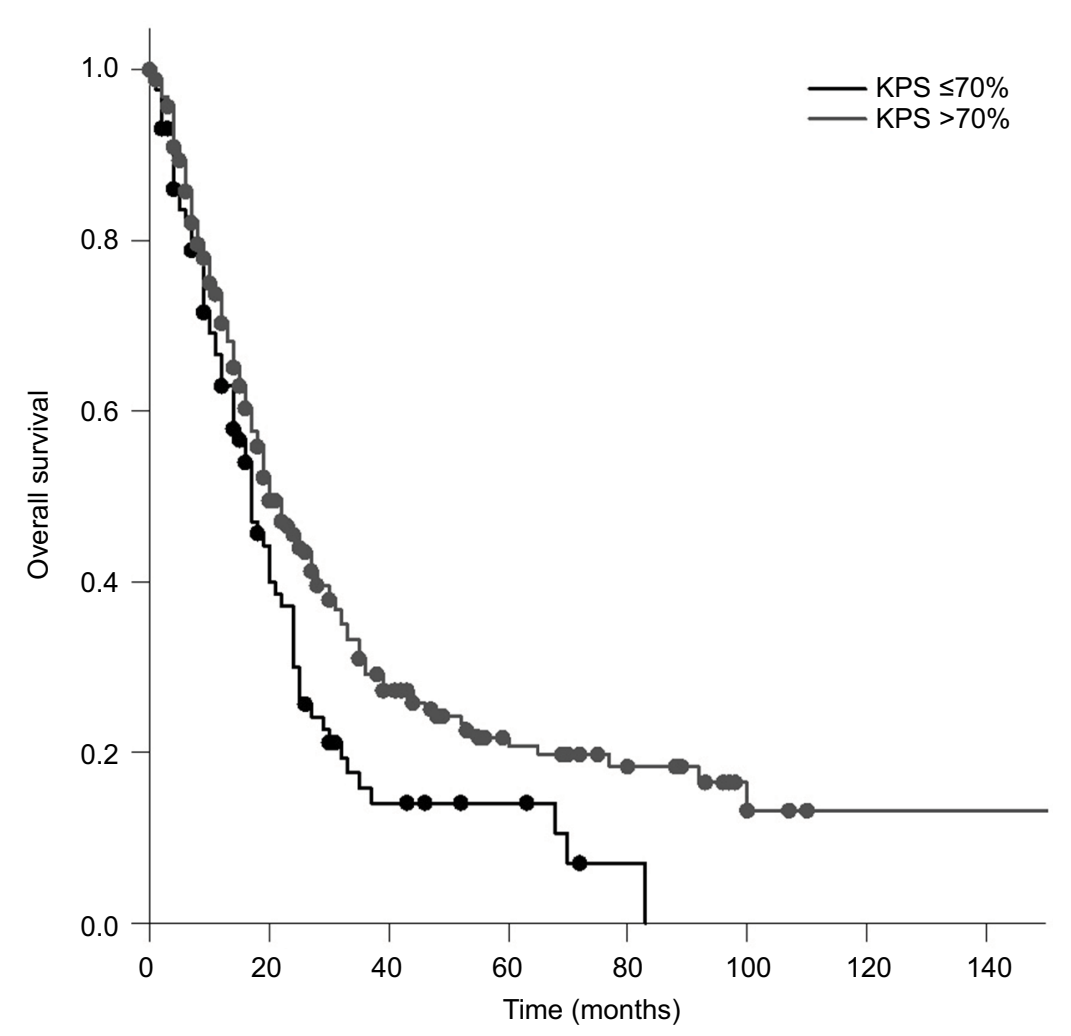

Figure I OS in patients with a KPS $\leq 70 \%$ compared to that in patients with a KPS $>70 \%$ (Kaplan-Meier's estimation, time in months: 17 vs 20 months; $P=0.0$ I 0 ). Abbreviations: KPS, Karnofsky performance scale; OS, overall survival. 


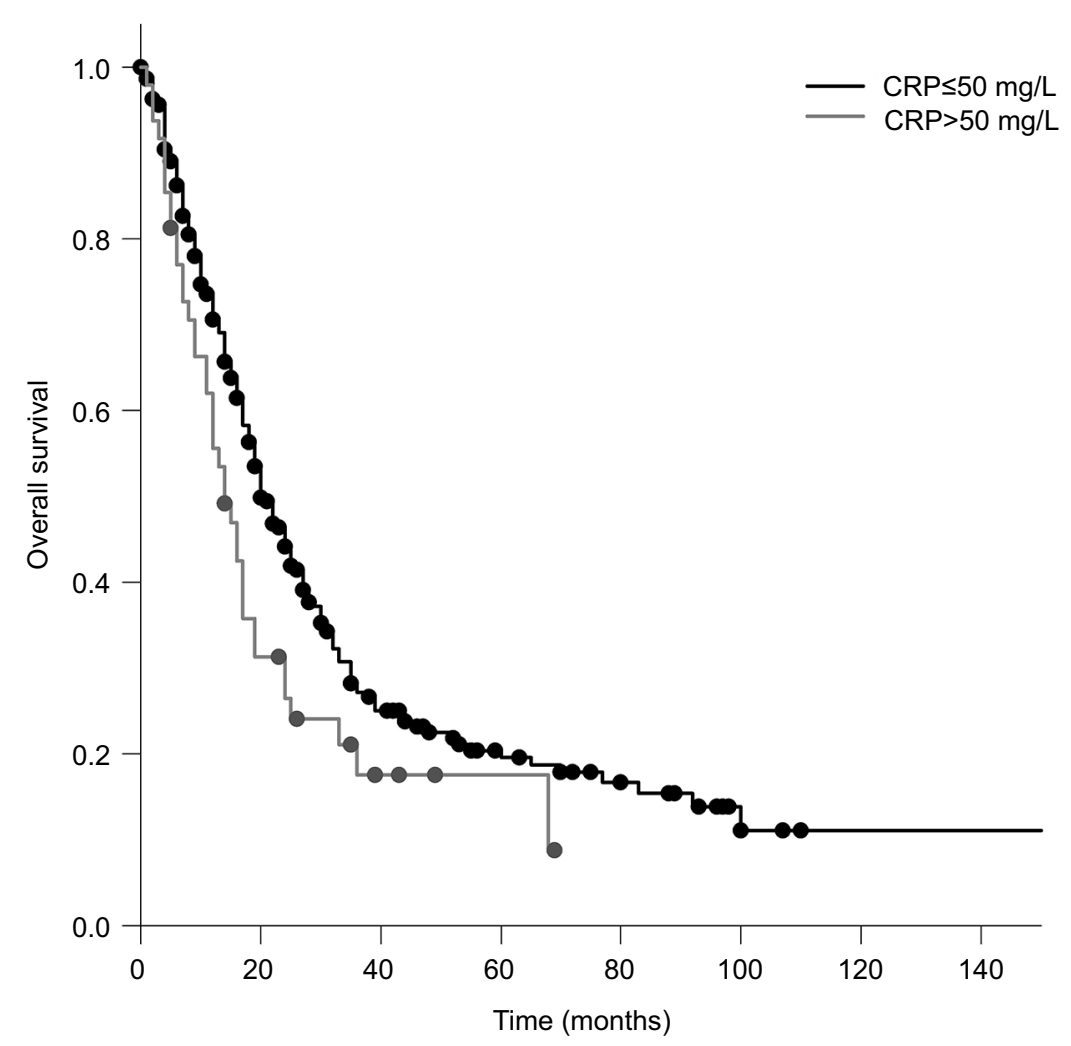

Figure 2 OS in patients with a CRP $\leq 50 \mathrm{mg} / \mathrm{L}$ compared to that in patients with a CRP $>50 \mathrm{mg} / \mathrm{L}$ (Kaplan-Meier's estimation, time in months: median 20 vs 14 months; $P=0.025$ ).

Abbreviations: CRP, C-reactive protein; OS, overall survival.

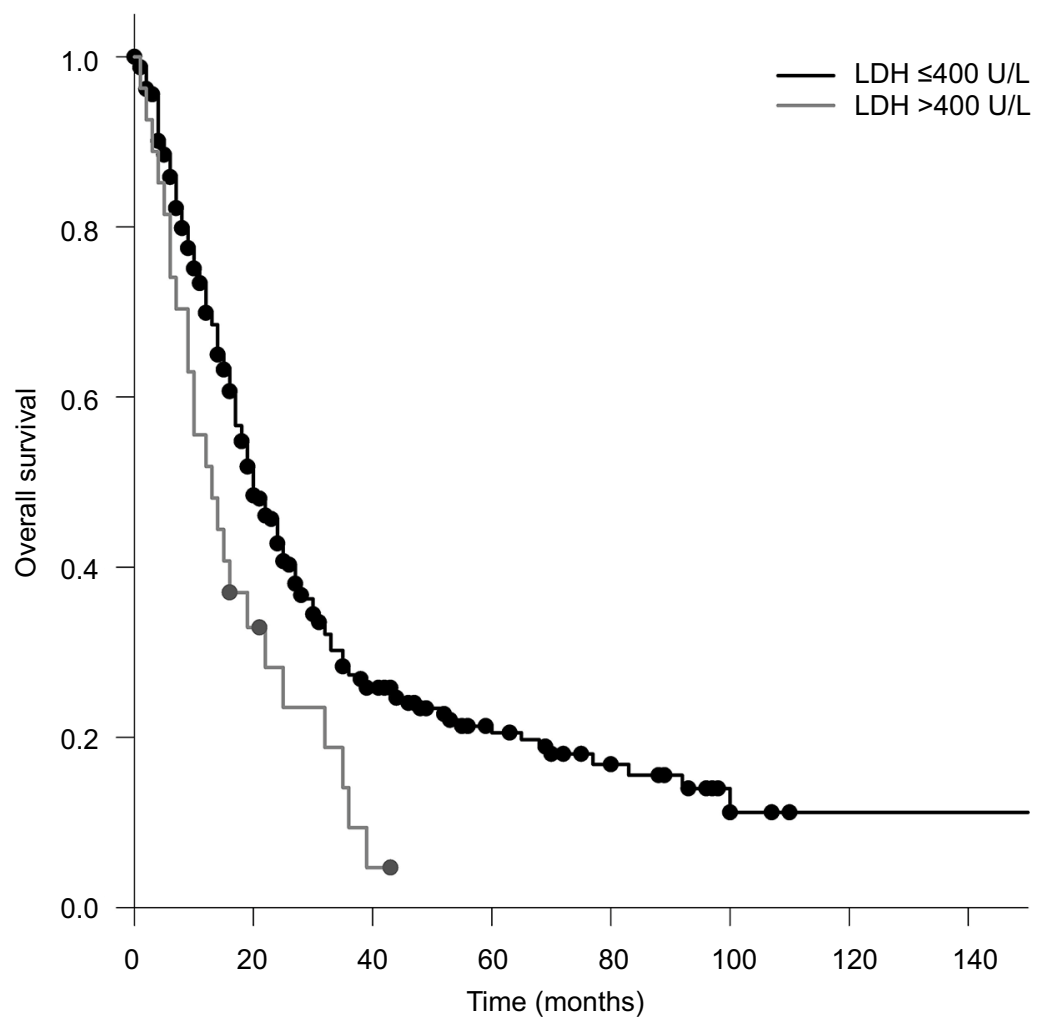

Figure 3 OS in patients with an $\mathrm{LDH}>400 \mathrm{U} / \mathrm{L}$ compared to that in patients with an $\mathrm{LDH} \leq 400 \mathrm{U} / \mathrm{L}$ (Kaplan-Meier's estimation, time in months: median 13 vs 20 months; $P=0.008$ ).

Abbreviations: LDH, lactate dehydrogenase; OS, overall survival. 
factors, such as CRP, NLR, LDH, and Pc, have been studied in various types of solid tumors and have independent prognostic values across tumor types in patients with advanced cancer. ${ }^{713,24,27-31}$ Furthermore, NLR is an inexpensive, easy accessible biomarker that has the potential to be helpful with clinical decision making and stratify patients in clinical trials. Several studies also showed the potential benefit of inflammatory markers as prognostic factors in lung cancer patients. A recent meta-analysis revealed that a high NLR had an unfavorable effect on NSCLC patients. A subgroup analysis showed that, compared with other subgroups, the subgroup with a cutoff of 5 had a significantly poorer survival in NSCLC patients. ${ }^{7}$ Furthermore, another meta-analysis demonstrated that elevated pretreatment NLR was associated with poor prognosis among patients with lung cancer. ${ }^{32}$ Potentially prognostic markers can also serve as indexes for the immune status of the patients and the extent of progression, ${ }^{17,18}$ and furthermore, these markers were shown to be predictive of the therapeutic efficacy of immunotherapy. ${ }^{8,17}$ In a previous study, NSCLC patients were treated with nivolumab, and an NLR $\geq 5$ prior to therapy was associated with shorter OS. Additionally, patients with lower serum CRP levels had a significantly longer median time to treatment failure. ${ }^{17}$ In a prospective observational study, NLR was found to be a potential candidate for a convenient biomarker regardless of PD-L1 expression. ${ }^{12}$

A previous study showed that NLR can be an independent predictor of OS in LD SCLC as an NLR of $\geq 4$ resulted in significantly shorter survival than an NLR of $<4 .{ }^{20}$ Similar results were published by a Korean group, who also showed that an NLR of $>4$ and elevated LDH were associated with poor prognosis. Platelet-lymphocyte ratio (PLR) at diagnosis was not associated with OS. ${ }^{33}$ However, the number of patients was relatively low, or LD and extensive disease (ED) patients were grouped together ( $n=64$ and $n=187$, respectively). A Chinese study showed that high NLR $>4.55$ and PLR $>148$ were associated with poor overall prognosis and high NLR value, ED stage, and hepatic metastasis were independent prognostic factors in LD and ED SCLC. ${ }^{34}$ A large and well-selected retrospective study conducted at the MD Anderson Center investigated the effect of NLR in ED patients $(n=252)$ and found that pretreatment NLR was a useful predictor of outcome in patients treated for ED SCLC. Comparable to the present study, pretreatment Pc was not linked with OS. ${ }^{35}$ Another study revealed not only an association between NLR and LDH and survival but also a link between NLR and platinum-sensitivity status. ${ }^{36}$ Deng et al ${ }^{11}$ observed in a mixed cohort of ED and LD patients that elevated NLR $>2.65$ before treatment was an independent prognostic factor of poor progression-free survival (PFS) and OS and LDH as an prognostic factor for OS and PFS.

In contrast to our study, several trials were able to show a prognostic value of NLR in lung cancer patients. Currently, there are no defined cutoff values, and several reports have used different cutoff values when evaluating the prognostic value of NLR. ${ }^{6}$ We tried to address this issue by using different and previously used cutoff values. Nevertheless, NLR was not found to be prognostic for OS in this cohort. Inflammatory comorbidities, infections, and steroid treatments could be confounding factors. In addition, the timing of blood count might be relevant, as we assessed NLR within 2 weeks prior to the start of chemotherapy because NLR might have been a useful tool for pretherapy stratification of patients. Perhaps, NLR might be useful in prospective oncology monitoring with several time points, but using an NLR cutoff value for clinical decision making prior to any therapy requires extreme caution in SCLC patients. Comparable to NSCLC patients, NLR might be a prognostic marker for SCLC patients undergoing second-line immunotherapy, though currently there are no data available. ${ }^{37}$

This study was limited by its retrospective nature, but we tried to address these biases by presenting a large, wellselected, and homogeneous group of patients.

\section{Conclusion}

Our findings suggest that pretreatment-elevated CRP and LDH are prognostic factors for patients with LD SCLC undergoing TCR. LDH and CRP are easy to use and costeffective. In contrast to previous reports, NLR was not associated with survival measured before the start of TCR. Therefore, the use of NLR as a prognostic tool for treatment stratification prior to therapy in LD SCLC patients must be viewed with caution. Further research is needed to investigate the role of inflammatory markers in SCLC patients undergoing immunotherapy.

\section{Acknowledgments}

This work was supported by Heidelberg University Young Investigator Grants to DB, LK, RAES, and JHR.

An abstract of this paper was presented at the 24th Annual Meeting of the German Society of Radiation Oncology; June 21-24, 2018, Leipzig, Germany. The poster's abstract has been published. ${ }^{38}$

\section{Disclosure}

JD reports grants and personal fees from Merck Serono, outside the submitted work. The authors report no other conflicts of interest in this work. 


\section{References}

1. Singel KL, Segal BH. Neutrophils in the tumor microenvironment: trying to heal the wound that cannot heal. Immunol Rev. 2016;273(1): 329-343.

2. Moses K, Brandau S. Human neutrophils: Their role in cancer and relation to myeloid-derived suppressor cells. Semin Immunol. 2016;28(2):187-196.

3. Pinkerton JW, Kim RY, Robertson AAB, et al. Inflammasomes in the lung. Mol Immunol. 2017;86:44-55.

4. Hanahan D, Weinberg RA. Hallmarks of cancer: the next generation. Cell. 2011;144(5):646-674.

5. Kim EY, Kim N, Kim YS, et al. Prognostic Significance of Modified Advanced Lung Cancer Inflammation Index (ALI) in Patients with Small Cell Lung Cancer_Comparison with Original ALI. PLoS One. 2016;11(10):e0164056.

6. Vano YA, Oudard S, By MA, et al. Optimal cut-off for neutrophilto-lymphocyte ratio: Fact or Fantasy? A prospective cohort study in metastatic cancer patients. PLoS One. 2018;13(4):e0195042.

7. Peng B, Wang YH, Liu YM, Ma LX. Prognostic significance of the neutrophil to lymphocyte ratio in patients with non-small cell lung cancer: a systemic review and meta-analysis. Int J Clin Exp Med. 2015;8(3):3098-3106.

8. Sacdalan DB, Lucero JA, Sacdalan DL. Prognostic utility of baseline neutrophil-to-lymphocyte ratio in patients receiving immune checkpoint inhibitors: a review and meta-analysis. Onco Targets Ther. 2018;11:955-965.

9. Kiriu T, Yamamoto M, Nagano T, et al. The time-series behavior of neutrophil-to-lymphocyte ratio is useful as a predictive marker in nonsmall cell lung cancer. PLoS One. 2018;13(2):e0193018.

10. Wang X, Teng F, Kong L, Yu J. Pretreatment neutrophil-to-lymphocyte ratio as a survival predictor for small-cell lung cancer. Onco Targets Ther. 2016;9:5761-5770.

11. Deng M, Ma X, Liang X, Zhu C, Wang M. Are pretreatment neutrophillymphocyte ratio and platelet-lymphocyte ratio useful in predicting the outcomes of patients with small-cell lung cancer? Oncotarget. 2017;8(23):37200-37207.

12. Fukui T, Okuma Y, Nakahara Y, et al. Activity of Nivolumab and Utility of Neutrophil-to-Lymphocyte Ratio as a Predictive Biomarker for Advanced Non-Small-Cell Lung Cancer: A Prospective Observational Study. Clin Lung Cancer. Epub 2018 May 5.

13. Bowen RC, Little NAB, Harmer JR, et al. Neutrophil-to-lymphocyte ratio as prognostic indicator in gastrointestinal cancers: a systematic review and meta-analysis. Oncotarget. 2017;8(19):32171-32189.

14. Zhang H, Zhang L, Zhu K, et al. Prognostic Significance of Combination of Preoperative Platelet Count and Neutrophil-Lymphocyte Ratio (COP-NLR) in Patients with Non-Small Cell Lung Cancer: Based on a Large Cohort Study. PLoS One. 2015;10(5):e0126496.

15. Yang HB, Xing M, Ma LN, Feng LX, Yu Z. Prognostic significance of neutrophil-lymphocyte ratio/platelet-lymphocyte ratio in lung cancers: a meta-analysis. Oncotarget. 2016;7(47):76769-76778.

16. Cannon NA, Meyer J, Iyengar P, et al. Neutrophil-lymphocyte and platelet-lymphocyte ratios as prognostic factors after stereotactic radiation therapy for early-stage non-small-cell lung cancer. JThorac Oncol. 2015;10(2):280-285.

17. Akamine T, Takada K, Toyokawa G, et al. Association of preoperative serum CRP with PD-L1 expression in 508 patients with non-small cell lung cancer: A comprehensive analysis of systemic inflammatory markers. Surg Oncol. 2018;27(1):88-94.

18. Zer A, Sung MR, Walia P, et al. Correlation of Neutrophil to Lymphocyte Ratio and Absolute Neutrophil Count With Outcomes With PD-1 Axis Inhibitors in Patients With Advanced Non-Small-Cell Lung Cancer. Clin Lung Cancer. 2018;19(5):426-434.e1.
19. Zelen M. Keynote address on biostatistics and data retrieval. Cancer Chemother Rep 3. 1973;4(2):31-42.

20. Käsmann L, Bolm L, Schild SE, Janssen S, Rades D. Neutrophil-toLymphocyte Ratio Predicts Outcome in Limited Disease Small-cell Lung Cancer. Lung. 2017;195(2):217-224.

21. Giraldo NA, Becht E, Vano Y, Sautès-Fridman C, Fridman WH. The immune response in cancer: from immunology to pathology to immunotherapy. Virchows Arch. 2015;467(2):127-135.

22. Shrotriya S, Walsh D, Bennani-Baiti N, Thomas S, Lorton C. C-Reactive Protein Is an Important Biomarker for Prognosis Tumor Recurrence and Treatment Response in Adult Solid Tumors: A Systematic Review. PLoS One. 2015;10(12):e0143080.

23. Leuzzi G, Galeone C, Gisabella M, et al. Baseline C-reactive protein level predicts survival of early-stage lung cancer: evidence from a systematic review and meta-analysis. Tumori. 2016;102(5):441-449.

24. Dolan RD, McSorley ST, Horgan PG, Laird B, McMillan DC. The role of the systemic inflammatory response in predicting outcomes in patients with advanced inoperable cancer: Systematic review and meta-analysis. Crit Rev Oncol Hematol. 2017;116:134-146.

25. Fang $\mathrm{Y}, \mathrm{Xu} \mathrm{C}, \mathrm{Wu} \mathrm{P}$, et al. Prognostic role of $\mathrm{C}$-reactive protein in patients with nasopharyngeal carcinoma. Medicine (Baltimore). 2017;96(45):e8463.

26. Grose D, Morrison DS, Devereux G, et al; Scottish Lung Cancer Forum. The impact of comorbidity upon determinants of outcome in patients with lung cancer. Lung Cancer. 2015;87(2):186-192.

27. Zhou Y, Cheng S, Fathy AH, Qian H, Zhao Y. Prognostic value of platelet-to-lymphocyte ratio in pancreatic cancer: a comprehensive metaanalysis of 17 cohort studies. Onco Targets Ther. 2018;11:1899-1908.

28. Catacchio I, Scattone A, Silvestris N, Mangia A. Immune Prophets of Lung Cancer: The Prognostic and Predictive Landscape of Cellular and Molecular Immune Markers. Transl Oncol. 2018;11(3):825-835.

29. Tham T, Bardash Y, Herman SW, Costantino PD. Neutrophil-tolymphocyte ratio as a prognostic indicator in head and neck cancer: A systematic review and meta-analysis. Head Neck. Epub 2018 May 14.

30. Wang Y, Peng C, Cheng Z, et al. The prognostic significance of preoperative neutrophil-lymphocyte ratio in patients with hepatocellular carcinoma receiving hepatectomy: A systematic review and meta-analysis. Int J Surg. 2018;55:73-80.

31. Zhan H, Ma JY, Jian QC. Prognostic significance of pretreatment neutrophil-to-lymphocyte ratio in melanoma patients: A meta-analysis. Clin Chim Acta. 2018;484:136-140.

32. Yu Y, Qian L, Cui J. Value of neutrophil-to-lymphocyte ratio for predicting lung cancer prognosis: A meta-analysis of 7,219 patients. Mol Clin Oncol. 2017;7(3):498-506.

33. Kang MH, Go SI, Song HN, et al. The prognostic impact of the neutrophil-to-lymphocyte ratio in patients with small-cell lung cancer. Br J Cancer. 2014;111(3):452-460.

34. Liu D, Huang Y, Li L, Song J, Zhang L, Li W. High neutrophil-tolymphocyte ratios confer poor prognoses in patients with small cell lung cancer. BMC Cancer. 2017;17(1):882.

35. Suzuki R, Lin SH, Wei X, et al. Prognostic significance of pretreatment total lymphocyte count and neutrophil-to-lymphocyte ratio in extensivestage small-cell lung cancer. Radiother Oncol. 2018;126(3):499-505.

36. Wen Q, Meng X, Xie P, Wang S, Sun X, Yu J. Evaluation of factors associated with platinum-sensitivity status and survival in limitedstage small cell lung cancer patients treated with chemoradiotherapy. Oncotarget. 2017;8(46):81405-81418.

37. Antonia SJ, López-Martin JA, Bendell J, et al. Nivolumab alone and nivolumab plus ipilimumab in recurrent small-cell lung cancer (CheckMate 032): a multicentre, open-label, phase 1/2 trial. Lancet Oncol. 2016;17(7):883-895.

38. [No authors listed]. Abstracts DEGRO 2018. Strahlenther Onkol. 2018;194(Suppl 1):1-222. 
Cancer Management and Research

\section{Publish your work in this journal}

Cancer Management and Research is an international, peer-reviewed open access journal focusing on cancer research and the optimal use of preventative and integrated treatment interventions to achieve improved outcomes, enhanced survival and quality of life for the cancer patient.

The manuscript management system is completely online and includes

Submit your manuscript here: https://www.dovepress.com/cancer-management-and-research-journal 